\title{
DESENVOLVIMENTO E VALIDAÇÃO DE UM MÉTODO ANALÍTICO RÁPIDO POR CROMATOGRAFIA LÍQUIDA DE ALTA EFICIÊNCIA PARA DETERMINAÇÃO DE NIMESULIDA EM ESTUDOS DE LIBERAÇÃO IN VITRO
}

\author{
André Luís Morais Ruela, Magali Benjamim Araújo e Gislaine Ribeiro Pereira* \\ Departamento de Farmácia, Universidade Federal de Alfenas, Rua Gabriel Monteiro da Silva, 714, 37130-000 Alfenas - MG, \\ Brasil
}

Recebido em 6/6/08; aceito em 18/8/08; publicado na web em 15/12/08

\begin{abstract}
DEVELOPMENT AND VALIDATION OF A RAPID ANALYTICAL METHOD BY HPLC FOR DETERMINATION OF NIMESULIDE IN RELEASE STUDIES. A high performance liquid chromatography (HPLC) method has been developed for a rapid determination of nimesulide in dissolution studies. Nimesulide was analyzed using $5 \mu \mathrm{m}$ Lichrospher® RP-18 column (125 x $4 \mathrm{~mm}$ i.d.) and mobile phase acetonitrile: phosphate buffer $\mathrm{pH}=6.0(55: 45)$ at a flow-rate of $1.0 \mathrm{~mL} \mathrm{~min}^{-1}$. Detection was carried out at $300 \mathrm{~nm}$ at $25^{\circ} \mathrm{C}$. The method was applied to analysis of nimesulide in in vitro release studies and showed a rapid and efficient analytical alternative for evaluation of dissolution profile of nimesulide.
\end{abstract}

Keywords: nimesulide; high performance liquid chromatography; dissolution study.

\section{INTRODUÇÃO}

A nimesulida ( $N$-(4-nitro-2-fenoxifenil)metanossulfonamida) é um antiinflamatório não-esteróide inibidor seletivo da enzima ciclooxigenase (COX) do tipo 2. ${ }^{1}$ Apresenta-se como um ácido fraco praticamente insolúvel em água, pouco solúvel em etanol e facilmente solúvel em acetona. ${ }^{2}$ Quimicamente, o fármaco é uma alquilssulfonamida, considerada protótipo da classe das metassulfonamidas, principalmente devido à potência antiinflamatória e ao perfil de segurança terapêutico. ${ }^{3} \mathrm{~A}$ estrutura química da nimesulida está apresentada na Figura 1.<smiles>CS(=O)(=O)Nc1ccc([N+](=O)[O-])cc1Oc1ccccc1</smiles>

Figura 1. Estrutura química da nimesulida

O mecanismo de ação da nimesulida baseia-se na inibição da COX-2, decorrente da interação do grupamento sulfonamida da molécula do fármaco com um resíduo de arginina em uma cavidade hidrofílica da enzima. O fármaco é amplamente comercializado atualmente em formas farmacêuticas orais, como comprimidos, comprimidos dispersíveis, gotas, granulados e suspensões, pois estas reduzem a incidência de efeitos indesejáveis associados à inibição da COX tipo 1 fisiológica, como as irritações gastrointestinais. ${ }^{4,5}$

Em uma revisão recente, a cromatografia líquida de alta eficiência (CLAE) foi a técnica analítica mais empregada na determinação de

\footnotetext{
*e-mail: gribeiro@unifal-mg.edu.br
}

inibidores da COX-2 em diferentes matrizes, seguida pela espectrofotometria na região do ultravioleta e visível. De acordo com o mesmo trabalho, pôde-se perceber que as metodologias apresentam uma grande variação nas condições cromatográficas empregadas, principalmente no que se refere à detecção, composição e $\mathrm{pH}$ da fase móvel e tipo de coluna utilizada. ${ }^{5}$

Estudos para determinação de nimesulida por CLAE em matrizes biológicas (fluido cerebrospinal, plasma e humor aquoso) são desenvolvidos geralmente com corridas mais longas para assegurar a eficiência de separação e são validados para faixas de trabalho limitadas..$^{6-9}$ Em um estudo para determinação de nimesulida e seus principais metabólitos em urina, usou-se método de eluição por gradiente e as corridas foram realizadas em $50 \mathrm{~min} .{ }^{10}$ Outros estudos por CLAE desenvolveram metodologias para determinação do fármaco em presença de produtos de degradação ou misturas com outros fármacos. ${ }^{11,12}$ Tais métodos apresentaram faixas de trabalho mais amplas, porém a necessidade de obter uma separação adequada para quantificar outros compostos exigiu corridas mais longas para detecção da nimesulida. Em um estudo para quantificação de impurezas, a realização de corridas mais curtas foi possível devido ao emprego de uma coluna do tipo monolítica e vazões de fase móvel elevadas. ${ }^{13}$

$\mathrm{O}$ interesse crescente em métodos rápidos por CLAE para quantificação de fármacos deve-se ao fato de que os mesmos podem ser aplicados a diferentes estudos, como os ensaios de dissolução. Atualmente, o emprego de métodos por CLAE para quantificação do fármaco em estudos de dissolução vem aumentando, uma vez que a dissolução in vitro do princípio ativo é um importante parâmetro para as indústrias farmacêuticas em diferentes estágios das etapas de desenvolvimento e produção de um medicamento. ${ }^{14}$

Entretanto, o emprego de um método analítico por CLAE em estudos de dissolução exige corridas curtas, pois para avaliação do perfil de dissolução, em que é construída uma curva da porcentagem do medicamento dissolvido ao longo do tempo, gera-se uma grande quantidade de amostras. No caso de fármacos pouco solúveis, além da rapidez, o método deve considerar a interferência de tensoativos durante a análise..$^{15,16}$ Os tensoativos ou surfactantes, como lauril sulfato de sódio e o polissorbato 80, são comumente acrescidos aos meios de dissolução como estratégia para aumentar a solubilidade em 
água do fármaco insolúvel, de modo a garantir condições sink e tornar o estudo de dissolução mais próximo das condições fisiológicas. ${ }^{17}$

Atualmente, há grande interesse no desenvolvimento de métodos analíticos rápidos e que forneçam parâmetros apropriados para análises quantitativas de fármacos. Tais métodos são importantes para análises de rotina durante o controle de qualidade e também no desenvolvimento de novas formas farmacêuticas. Diante disso, propõe-se uma metodologia rápida para análise de comprimidos de nimesulida em estudos de liberação in vitro, uma vez que ainda não foi descrito um método por CLAE empregando colunas de fase reversa apropriado para análise do fármaco em estudos de dissolução.

\section{PARTE EXPERIMENTAL}

\section{Materiais}

\section{Produtos farmacêuticos, padrão de referência e solventes}

Nimesulida padrão primário (100,0\%, Farmacopéia Européia, Lote 1a). Comprimidos de nimesulida $100 \mathrm{mg}$ de dois fabricantes diferentes (produtos 1 e 2) adquiridos no mercado nacional. Acetonitrila e metanol grau UV/HPLC. Os demais reagentes possuíam grau P.A.: ácido orto-fosfórico, fosfato de potássio monobásico anidro, fosfato de sódio monobásico anidro, hidróxido de sódio e polissorbato 80 (poli 80 ).

\section{Equipamentos}

Cromatógrafo a líquido (Shimadzu, Japão) com detector ultravioleta SPD-10AVP. Cromatógrafo a líquido (Shimadzu, Japão) com detector de arranjo de diodos SPD-M10AVP. Coluna cromatográfica LiChrospher ${ }^{\circledR}$ RP-select B (Merck, Alemanha) com 12,5 cm de comprimento, 4,0 $\mathrm{mm}$ d.i., $5 \mu \mathrm{m}$ de tamanho de partícula e pré-coluna LiChrospher ${ }^{\circledR}$ RP-select B (Merck, Alemanha) com 4 cm de comprimento, 4,0 mm de d.i. e $5 \mu \mathrm{m}$ de tamanho de partícula. Aparelho de ultrassom (Unique, 775 Brasil). Aparelho dissolutor de cubas 299/6 (Nova Ética, Brasil). Balança analítica (Kern, Alemanha). Espectrofotômetro duplo-feixe UV-1601 PC (Shimadzu, Japão). Medidor de pH modelo PA 200 (Marconi, Brasil).

\section{Metodologia}

\section{Parâmetros cromatográficos}

A fase móvel foi constituída de acetonitrila e tampão fosfato 20 $\mathrm{mM} \mathrm{pH} \mathrm{6,0} \mathrm{nas} \mathrm{proporções} \mathrm{de} \mathrm{55:45} \mathrm{v/v.} \mathrm{As} \mathrm{análises} \mathrm{foram} \mathrm{efetuadas}$ a $25{ }^{\circ} \mathrm{C}$ com vazão da fase móvel de $1,00 \mathrm{~mL} \mathrm{~min}^{-1}$. Realizaram-se corridas com 4 min de duração, empregando detecção no ultravioleta a $300 \mathrm{~nm}$. O volume de injeção foi de $20 \mu \mathrm{L}$.

\section{Validação do método analítico}

Os ensaios para determinação dos parâmetros de validação foram realizados de acordo com recomendações da legislação vigente na resolução RE 899 da Agência Nacional de Vigilância Sanitária (ANVISA).$^{18}$ As amostras foram preparadas em tampão fosfato $50 \mathrm{mM}$ pH 7,40 + 1,0\% (m/v) de poli 80, usado como meio de dissolução. Todas as soluções foram previamente filtradas em filtros de $0,45 \mu \mathrm{m}$ antes da injeção.

A pureza do pico cromatográfico foi determinada em cromatógrafo acoplado a detector de arranjo de diodos (DAD). Para isso, foram injetadas soluções da substância química de referência (padrão primário) e da forma farmacêutica comprimidos (produto 1) nas concentrações de $100 \mu \mathrm{g} \mathrm{mL}^{-1}$ e realizadas varreduras espectrais.

O estudo da linearidade foi realizado pela transferência de alíquotas de 1,$5 ; 3,0 ; 4,5 ; 6,0$ e 7,5 mL de solução estoque de nimesulida $500 \mu \mathrm{g} \mathrm{mL}^{-1}$ (preparada em acetonitrila) para balões volumétricos de $25 \mathrm{~mL}$ e o volume foi completado com o meio de dissolução. As concentrações obtidas foram de 30, 60, 90, 120 e $150 \mu \mathrm{g} \mathrm{mL}^{-1}$, respectivamente. As diluições foram preparadas em 5 réplicas e empregadas na construção da curva analítica concentração versus área do pico. A equação de reta e o coeficiente de correlação linear ( $r$ ) foram calculados.

A precisão intra-dias foi avaliada analisando-se soluções padrão $(\mathrm{n}=6)$ nas concentrações de 50 e $100 \mu \mathrm{g} \mathrm{mL}^{-1}$. As soluções foram preparadas pela transferência de alíquotas de $5,0 \mathrm{~mL}$ de solução estoque $500 \mu \mathrm{g} \mathrm{mL}^{-1}$ para balões volumétricos de 25 e $50 \mathrm{~mL}$, respectivamente, e o volume completado com o meio. As soluções foram analisadas e a seguir o coeficiente de variação $(\mathrm{CV} \%)$ foi calculado. A precisão inter-dias foi avaliada da mesma forma em dias consecutivos.

$\mathrm{O}$ estudo da exatidão foi realizado pela adição de substância química de referência à solução do produto 1 na concentração de $100,0 \mu \mathrm{g} \mathrm{mL}^{-1}$. Realizaram-se adições de 50, 100 e $150 \%$ de substância química de referência $(n=3)$. Após diluição apropriada dentro da faixa linear, realizaram-se as leituras e calculou-se o percentual de recuperação ( $\mathrm{R} \%)$.

Os limites de detecção e de quantificação (LD e LQ, respectivamente) foram calculados pela divisão do desvio padrão dos coeficientes lineares das 5 curvas de calibração do ensaio de linearidade pela média dos coeficientes angulares destas respectivas curvas, multiplicados por 3 e 10, respectivamente. Os resultados obtidos pelos cálculos foram analisados por injeções de soluções $(n=5)$ de concentrações decrescentes de 10,5, 1 e soluções correspondentes ao LQ e LD. Para confirmação do LQ, o coeficiente de correlação $(r)$ e o CV\% da concentração correspondente foram calculados.

A validação de uma metodologia apropriada para estudos de dissolução deve incluir outros parâmetros descritos na literatura. ${ }^{15}$ É importante a realização de um estudo do filtro e da estabilidade do fármaco no meio de dissolução. O estudo do filtro foi realizado pela análise de soluções padrão $100 \mu \mathrm{g} \mathrm{mL}^{-1}$ antes e após o processo de filtração. A estabilidade da nimesulida no meio de dissolução foi avaliada mantendo-se soluções padrão e amostra $100 \mu \mathrm{g} \mathrm{mL}^{-1}$ armazenadas ao abrigo de luz à temperatura ambiente $(n=3)$. Após $24 \mathrm{~h}$, realizaram-se leituras das mesmas soluções contra soluções padrão de nimesulida $100 \mu \mathrm{g} \mathrm{mL}^{-1}$ recém-preparadas $(\mathrm{n}=6)$ e determinou-se o percentual de resposta.

\section{Estudo de dissolução de comprimidos}

Realizou o estudo do perfil de dissolução da nimesulida a partir dos dois comprimidos comerciais (produtos 1 e 2) empregando-se $900 \mathrm{~mL}$ de meio tampão fosfato $50 \mathrm{mM} \mathrm{pH} \mathrm{7,40+1,0 \%} \mathrm{(m/v)} \mathrm{de}$ poli 80, aparato número 2 (pá) e rotação de $75 \mathrm{rpm}$. Alíquotas de $10,0 \mathrm{~mL}$ foram coletadas nos tempos de 5, 10, 15, 30 e $60 \mathrm{~min}$, em seguida filtradas e analisadas por CLAE. A partir dos resultados pôde-se construir a curva da porcentagem de nimesulida dissolvida versus tempo, que caracteriza o perfil de dissolução para os produtos avaliados.

\section{RESULTADOS E DISCUSSÃO}

O uso de metodologias por CLAE com coluna de fase reversa octadecilsilano é largamente empregado na determinação de fármacos. ${ }^{19}$ Deste modo, para análise da nimesulida selecionou-se a coluna de fase reversa Li-Chrospher ${ }^{\circledR}$ select-B. A composição da fase móvel foi definida após testar diferentes combinações de acetonitrila, metanol e água ou tampões aquosos. A fase móvel inicialmente testada foi acetonitrila e água (50:50), sendo que os resultados foram insatisfatórios devido à assimetria do pico cromatográfico. A simetria do pico melhorou quando se substituiu a água por tampão fosfato. As fases móveis com água são indicadas para compostos neutros, enquanto 
que para compostos ionizáveis como nimesulida é preferível o uso de tampões aquosos, uma vez que estes mantêm o grau de ionização das moléculas e evitam os problemas de retenção secundária, responsáveis pela formação de picos com caudas. ${ }^{19}$

A variação do $\mathrm{pH}$ da fase móvel permitiu o ajuste do tempo de retenção da nimesulida e, conseqüentemente, a realização de corridas mais curtas. Observou-se que o tempo de retenção é maior em $\mathrm{pH}$ ácido que em fases móveis com $\mathrm{pH}$ mais elevado, o que era esperado, pois, por se tratar de um ácido fraco de pKa 6.5, a nimesulida encontra-se predominantemente ionizada em valores de $\mathrm{pH}$ alcalinos e, desta forma, a interação do fármaco ionizado com a fase reversa é menor e o tempo de retenção provavelmente diminui. ${ }^{1} \mathrm{De}$ modo contrário, valores de $\mathrm{pH}$ ácidos favorecem a forma molecular do fármaco e aumentam a afinidade do mesmo por colunas de fase reversa, elevando o tempo de retenção. Em um trabalho em que foi desenvolvido um método rápido para análise de nimesulida em plasma humano, a fase móvel incluiu tampão fosfato $\mathrm{pH}$ 5,5 e o tempo de retenção do fármaco foi de $12,5 \mathrm{~min} .{ }^{8}$ Em outro trabalho para análise de nimesulida em plasma, a fase móvel possuía pH 3,0 e, no entanto, o tempo de retenção foi de 5,9 min, uma vez que foi empregada maior porcentagem de metanol (68\%) na fase móvel. ${ }^{9}$

A fase móvel acetonitrila:tampão fosfato $20 \mathrm{mM} \mathrm{pH} \mathrm{6,0} \mathrm{(55:45)}$ mostrou-se adequada para análise de nimesulida, o que incluiu um tempo de retenção reduzido (em torno de $3 \mathrm{~min}$ ), tornando o método aplicável em análises de rotina. A vazão de fase móvel e a temperatura da coluna foram mantidas em $1,0 \mathrm{~mL} \min ^{-1}$ e $25{ }^{\circ} \mathrm{C}$, respectivamente. A seleção do comprimento de onda foi realizada após varredura espectral na região do ultravioleta-visível (200-500 nm). Inicialmente, observou-se a maior absorção da nimesulida em uma banda de $200-230 \mathrm{~nm}$, entretanto, nesta faixa de comprimento de onda, o poli 80 usado para aumentar a solubilidade em água da nimesulida no meio de dissolução também apresentou forte absorção. Em corridas cromatográficas preliminares com detecção em $210 \mathrm{~nm}$, observou-se um pico referente ao poli $80 \mathrm{em}$ aproximadamente $10 \mathrm{~min}$. Deste modo, a detecção foi alterada para $300 \mathrm{~nm}$. Neste comprimento de onda, a nimesulida ainda apresentava um sinal analítico satisfatório para a análise e o poli 80 não foi detectado.

A Figura 2 apresenta o cromatograma de solução padrão de nimesulida $100 \mu \mathrm{g} \mathrm{mL}^{-1}$, utilizando fase móvel acetonitrila e tampão fosfato $\mathrm{pH}$ 6,0 (55:45) com detecção a $300 \mathrm{~nm}$.

A pureza do pico cromatográfico foi determinada após varredura do padrão e da amostra (solução de nimesulida a partir de comprimido) empregando detector de DAD. O detector de DAD é considerado

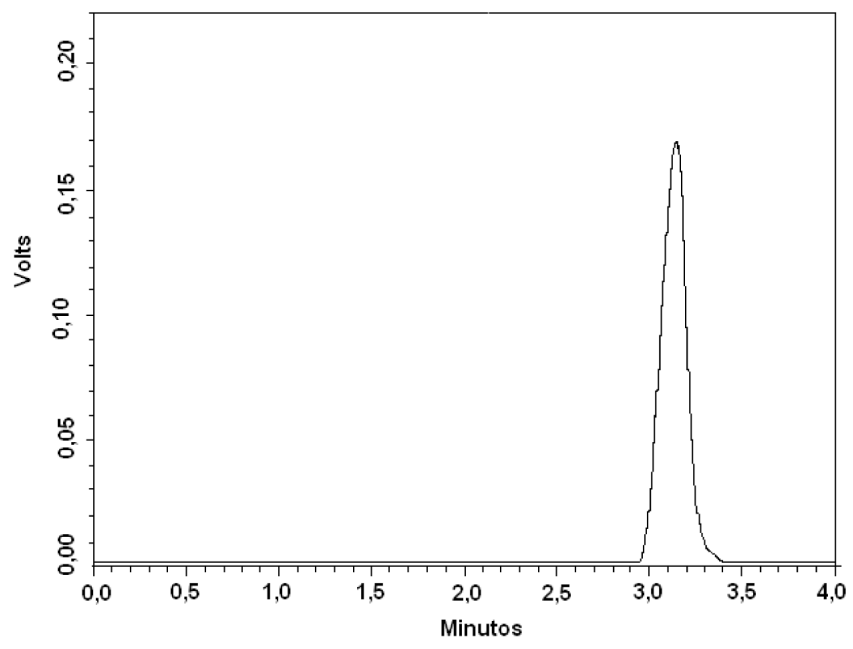

Figura 2. Perfil cromatográfico da nimesulida utilizando fase móvel acetonitrila e tampão fosfato $\mathrm{pH}$ 6,0 (55:45 v/v) com detecção a $300 \mathrm{~nm}$ uma precaução necessária para garantir a pureza do pico cromatográfico, em que o espectro do pico obtido na separação é comparado ao espectro de um padrão de alta pureza e o resultado é uma indicação da presença do composto puro na amostra. O percentual de resposta ao se comparar a área dos picos foi de $99,20 \%$.

A faixa linear foi selecionada de acordo com a concentração do fármaco no meio de dissolução se um comprimido de $100 \mathrm{mg}$ estiver totalmente dissolvido em $900 \mathrm{~mL}$ do meio $\left(111 \mu \mathrm{g} \mathrm{mL}^{-1}\right)$. A linearidade do método foi observada na faixa avaliada de $30 \mathrm{a} 150 \mu \mathrm{g} \mathrm{mL}^{-1}$. A equação de reta foi $y=14321 x-10057$ e o $r=0,9996$.

A Tabela 1 apresenta os valores referentes à avaliação da precisão e exatidão. Os resultados apresentados estão de acordo com as recomendações da ANVISA. ${ }^{18}$

Tabela 1. Avaliação da precisão e exatidão do método para análise de nimesulida por CLAE

\begin{tabular}{|c|c|c|c|}
\hline Parâmetros & \multicolumn{3}{|c|}{ Nimesulida } \\
\hline \multicolumn{4}{|c|}{ Precisão intra-ensaio $(\mathrm{n}=6)$} \\
\hline Concentração ( $\left.\mu \mathrm{g} \mathrm{mL}^{-1}\right)$ & 50,0 & & 100,0 \\
\hline $\mathrm{CV} \%$ & 0,17 & & 0,28 \\
\hline \multicolumn{4}{|c|}{ Precisão inter-ensaio $(\mathrm{n}=2)$} \\
\hline Concentração $\left(\mu \mathrm{g} \mathrm{mL}^{-1}\right)$ & 50,0 & & 100,0 \\
\hline $\mathrm{CV} \%$ & 0,73 & & 0,62 \\
\hline \multicolumn{4}{|l|}{ Exatidão (n=3) } \\
\hline Padrão Adicionado (\%) & 50 & 100 & 150 \\
\hline $\mathrm{R} \%$ & 98,75 & 99,64 & 98,04 \\
\hline
\end{tabular}

Os valores obtidos para o LD e o LQ de acordo com os cálculos

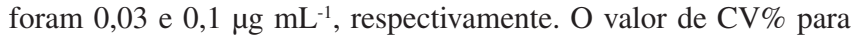
a concentração de $0,1 \mu \mathrm{g} \mathrm{mL} \mathrm{m}^{-1}$ foi de 2,6 e o $\mathrm{r}=0,9934$ para a faixa de 0,1 a $10,0 \mu \mathrm{g} \mathrm{mL}^{-1}$. Em estudos para amostras biológicas, que requerem faixas de trabalho mais sensíveis, o LQ encontrado foi de $0,05 \mu \mathrm{g} \mathrm{mL}$. $^{-7-9}$

A avaliação do filtro demonstrou que os resultados estão inseridos na faixa aceitável (98 a 102\%). O estudo é importante para demonstrar que o filtro não adsorve partículas de fármaco, nem libera material para solução. ${ }^{15}$ Após a filtração, a solução padrão apresentou percentual de resposta de $99,52 \%$.

A nimesulida apresentou-se estável no meio aquoso. A faixa aceitável de estabilidade é de 98 a 102\%. ${ }^{15}$ Os percentuais de resposta para as soluções de amostra e padrão foram de, respectivamente, 98,24 e $98,90 \%$.

A Figura 3 apresenta os perfis de dissolução de comprimidos de nimesulida em meio tampão fosfato $50 \mathrm{mM} \mathrm{pH} \mathrm{7,40+1,0 \%} \mathrm{(m/v)}$ de poli 80. O método analítico mostrou-se adequado para quantificação do fármaco durante estudos de liberação in vitro, permitindo a avaliação do perfil de dissolução dos produtos 1 e 2 . Na literatura, outros autores propuseram uma metodologia para análise do fármaco em testes de dissolução em meio tampão fosfato $\mathrm{pH} 7,40+$ poli 80 , entretanto, empregaram uma coluna monolítica e vazão de fase móvel de 4,0 mL min ${ }^{-1} \cdot{ }^{13}$ Os autores avaliaram a linearidade na faixa de 50 a $150 \mu \mathrm{g} \mathrm{mL}^{-1}$, o LQ e LD calculados foram de, respectivamente, 0,17 e $0,05 \mu \mathrm{g} \mathrm{mL}^{-1}$ e a precisão inter-dias igual a $1,2 \%{ }^{13}$

A proposição de um método rápido e de fácil execução é interessante para a rotina de controle de qualidade industrial. Deste modo, o método desenvolvido e validado para análise de nimesulida utilizando coluna de fase reversa atendeu às necessidades específicas do estudo de dissolução de comprimidos, mostrando-se uma alternativa analítica rápida e eficiente. 


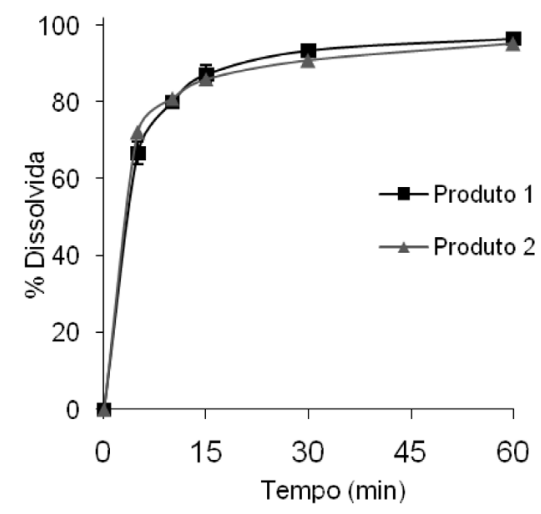

Figura 3. Perfil de dissolução dos produtos 1 e 2 em tampão fosfato $\mathrm{pH} 7,40$ $50 \mathrm{mM}+1,0 \%(\mathrm{~m} / \mathrm{v})$ de poli 80

\section{CONCLUSÃO}

A metodologia analítica empregando CLAE por fase reversa permitiu uma rápida detecção e quantificação da nimesulida. O método utilizando fase móvel constituída de acetonitrila:tampão fosfato $20 \mathrm{mM} \mathrm{pH} \mathrm{6,0} \mathrm{(55:45)} \mathrm{e} \mathrm{detecção} \mathrm{ultravioleta} \mathrm{a} 300 \mathrm{~nm}$ mostrou-se sensível ( $\mathrm{LQ}=0,1 \mu \mathrm{g} \mathrm{mL} \mathrm{mL}^{-1}$ ), preciso ( $\mathrm{CV} \%$ inferior a $1,0 \%$ ), exato (R\% de 98,08-99,64\%) e linear em ampla faixa de concentração $\left(0,1\right.$ a $\left.150 \mu \mathrm{g} \mathrm{mL}^{-1}\right)$. A pureza dos picos pôde ser determinada após varreduras espectrais empregando detector de DAD. Além disso, o método apresentou resultados satisfatórios para avaliação do perfil de dissolução de comprimidos de nimesulida, o que se mostrou interessante uma vez que não há metodologia oficial para avaliar a liberação do fármaco.

\section{AGRADECIMENTOS}

PIBIC/ CNPq e Unifal-MG.

\section{REFERÊNCIAS}

1. Dellis, D.; Giaginis, C.; Tsantili-Kakoulidou, A.; J. Pharm. Biomed. Anal. 2007, 44, 57.

2. Farmacopéia Portuguesa, $7^{\mathrm{a}}$ ed., Imprensa Nacional: Lisboa, 2002.

3. Leval, X.; Delarge, J.; Somers, F.; Tullio, P.; Henrotin, Y.; Pirotte, B.; Dogné, J.; Curr. Med. Chem. 2000, 7, 1041.

4. Lages, A. S.; Romeiro, N. C.; Fraga, C. A. M; Barreiro, E. J.; Quim. Nova 1998, 21, 761.

5. Rao, R. N.; Meena, S.; Rao, A. R.; J. Pharm. Biomed. Anal. 2005, 39, 349.

6. Ferrario, P.; Bianchi, M.; J. Chromatogr., B: Anal. Technol. Biomed. Life Sci. 2003, 785, 227.

7. Jaworowicz Jr, D. J.; Filipowski, M. T.; Boje, K. M. K.; J. Chromatogr., B: Anal. Technol. Biomed. Life Sci. 1999, 723, 293.

8. Khaksa, G.; Udupa, N.; J. Chromatogr., B: Anal. Technol. Biomed. Life Sci. 1999, 727, 241.

9. Maltese, A.; Maugeri, F.; Bucolo, C.; J. Chromatogr., B: Anal. Technol. Biomed. Life Sci. 2004, 804, 441.

10. Carini, M.; Aldini, G.; Stefani, R.; Marinello, C.; Facino, R. M.; J. Pharm. Biomed. Anal. 1998, 18, 201.

11. Kovarikova, P.; Mokry, M.; Klimes, J.; J. Pharm. Biomed. Anal. 2003, 31, 827.

12. Panusa, A.; Multari, G.; Incarnato, G.; Gagliardi, L.; J. Pharm. Biomed. Anal. 2007, 43, 1221.

13. Tzanavaras, P. D.; Themelis, D. G.; J. Pharm. Biomed. Anal. 2007, 43, 1483.

14. Aulton, M. E.; Delineamento de formas farmacêuticas, Artmed: Porto Alegre, 2005.

15. Marques, M. R. C.; Brown, W.; Rev. Analytica 2002, 1, 48.

16. Wang, Q.; Higgins, J. P.; Diss. Technol. 2006, 13, 6.

17. Park, S.; Choi, H.; Int. J. Pharm. 2006, 321, 35.

18. Agência Nacional de Vigilância Sanitária (ANVISA); Resolução $n^{\circ} R E$ 899, de 29 de maio de 2003

19. Harris, D. C.; Análise Química Quantitativa, 5ª ed., Livros Técnicos e Científicos: Rio de Janeiro, 2001. 